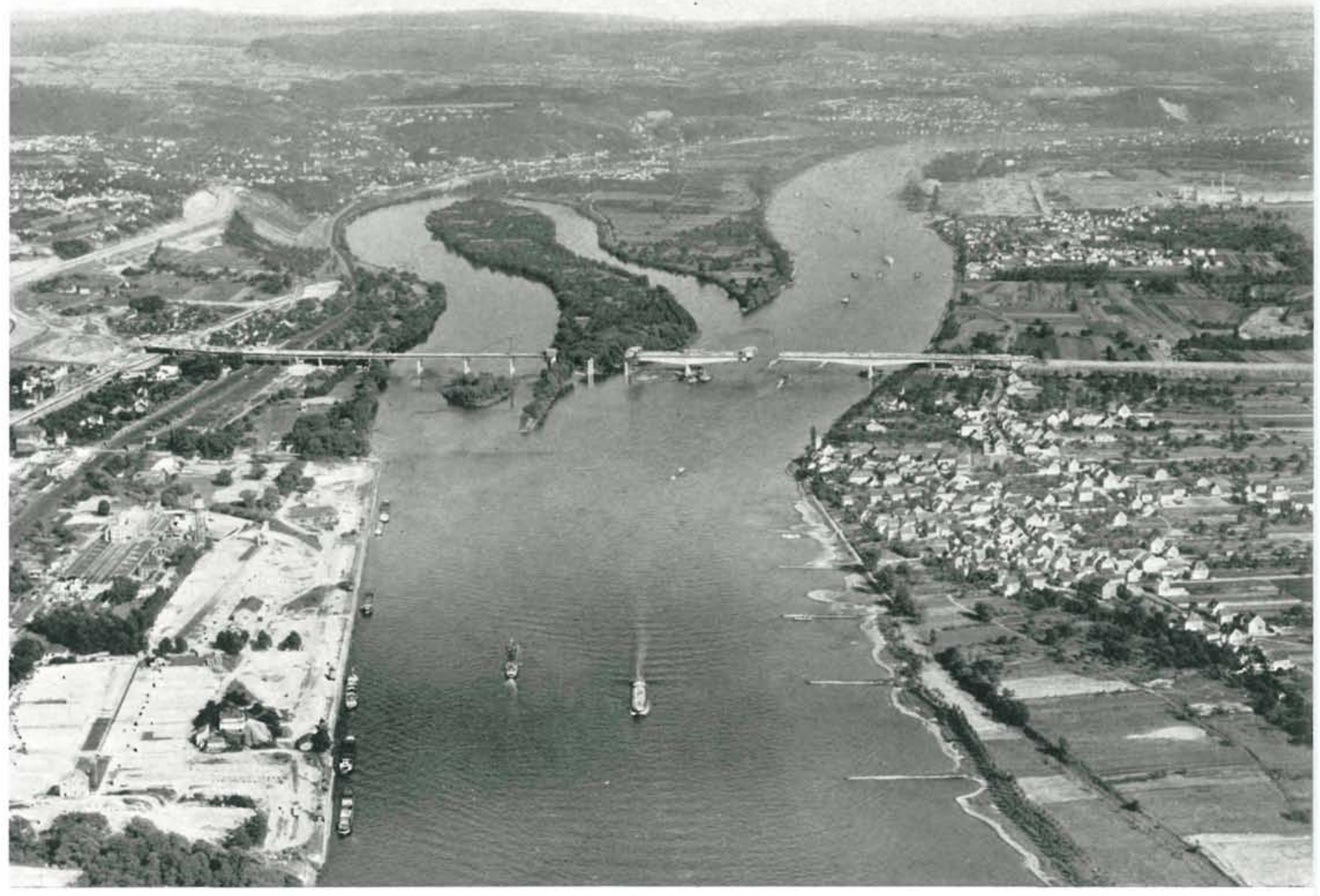

\title{
el puente Bendorf sobre el Rin ALEMANIA
}

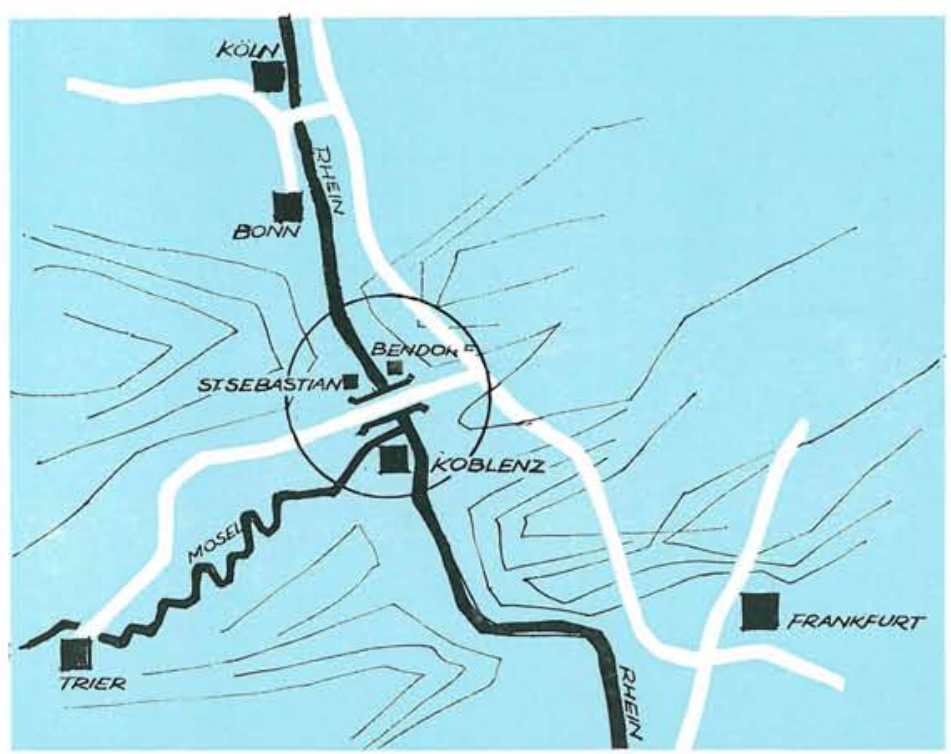

KURT HIRSCHFELD,

Prof. Dr. Ingeniero

$562-98$

sîn日 opsis

Este puente, recientemente construido, sobre el Rin, tiene una longitud total de $524 \mathrm{~m}$, es de hormigón armado $y$ se ha realizado por el procedimiento de voladizos Lucesivos, sin entramado auxiliar ni cim. Ios otros seis oscilan entre 43 y $71 \mathrm{~m}$. La estructura del tablero se compone de dos partes independientes, formadas por una viga tipo cajón, continua, con una articulación en la clave del tramo central y rigidizada con diafragmas transversales espaciados a $34 \mathrm{~m}$. Las pilas centrales se apoyan sobre cajones neumaticos que descienden $16 \mathrm{~m}$ debajo del fondo del río. La unión entre estas pilas y la estructura cajón es rígida, $y$ de tipo rodillo en las otras pilas. EI tramo central se ha construido por voladizos sucesivos, sin apoyo intermedio, en una longitud de $40 \mathrm{~m}$, a partir de la cual, y para mayo seguisial, se levanto un castillete hormigonaron y tesaron con harres, de 32 mos se dímetro, siguiendo los procedimientos Dywidag y teniendo en cuenta las distintas deformaciones expe. rimentadas en cada uno, a fin de introducir la contra. flecha necesaria que permitiera cerrar el tramo cen. tral a la cota prevista.

\section{siftuación}



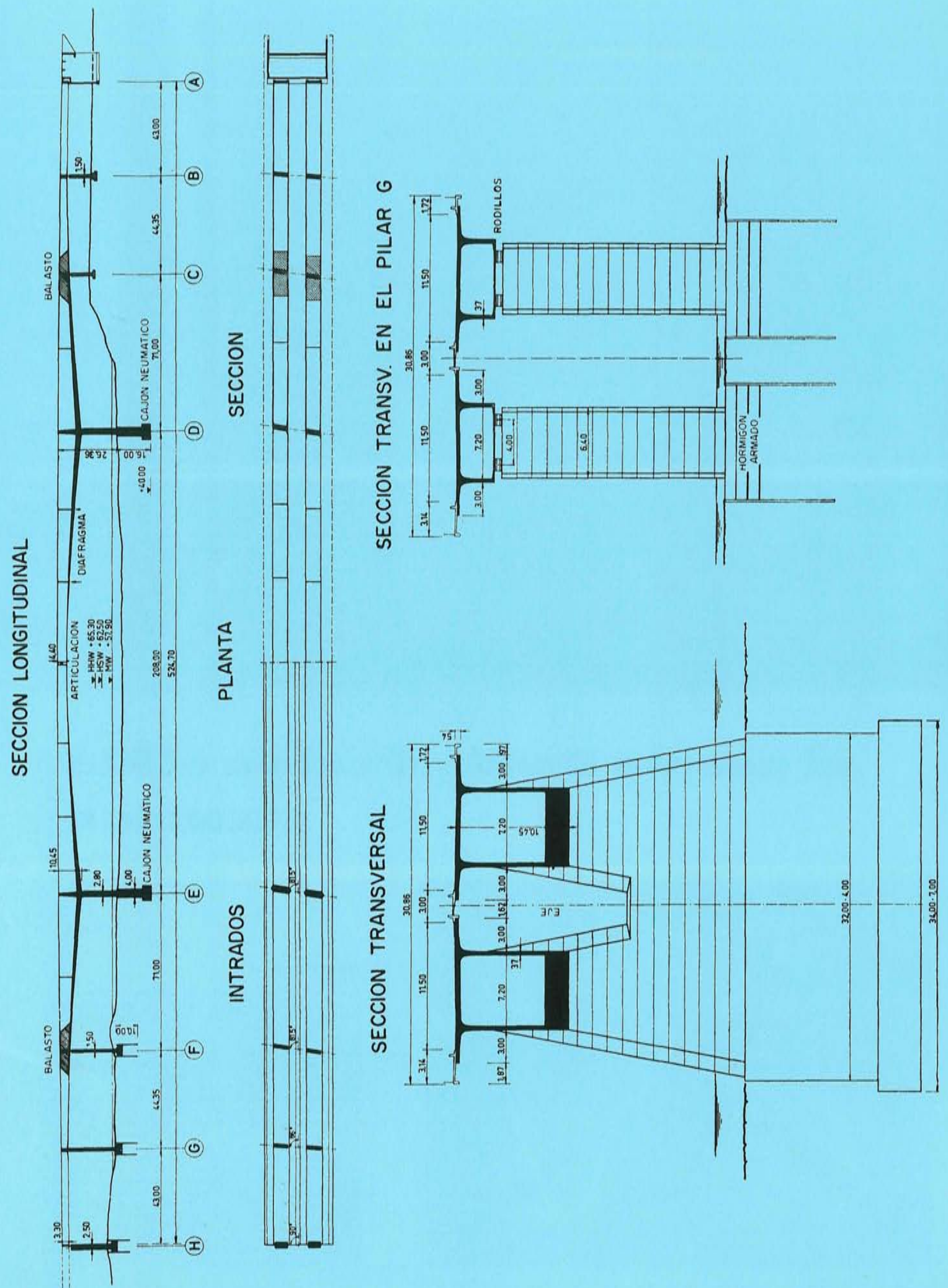
Estructura

cajón.
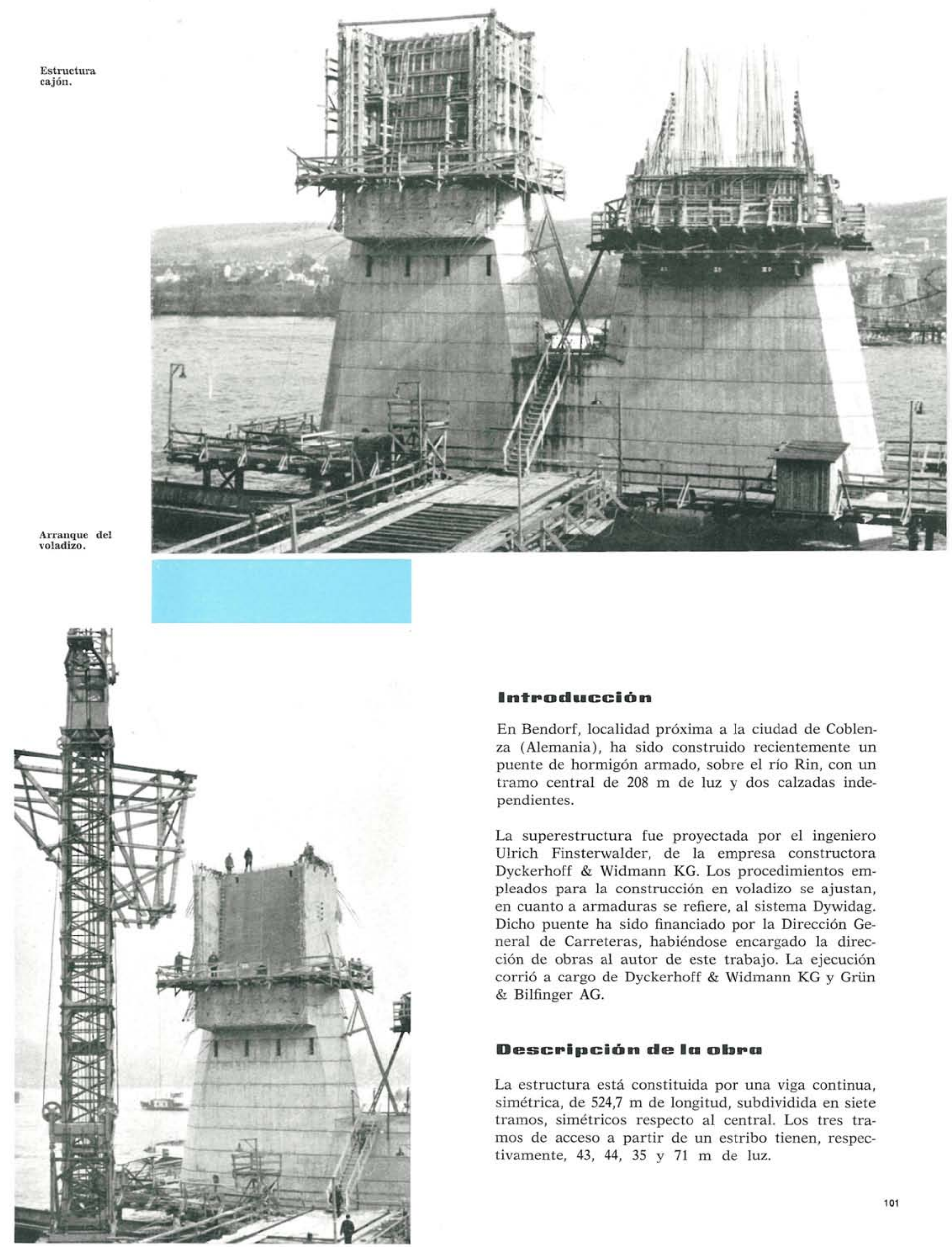

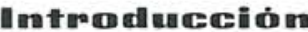

En Bendorf, localidad próxima a la ciudad de Coblenza (Alemania), ha sido construido recientemente un puente de hormigón armado, sobre el río Rin, con un tramo central de $208 \mathrm{~m}$ de luz y dos calzadas independientes.

La superestructura fue proyectada por el ingeniero Ulrich Finsterwalder, de la empresa constructora Dyckerhoff \& Widmann KG. Los procedimientos empleados para la construcción en voladizo se ajustan, en cuanto a armaduras se refiere, al sistema Dywidag. Dicho puente ha sido financiado por la Dirección General de Carreteras, habiéndose encargado la dirección de obras al autor de este trabajo. La ejecución corrió a cargo de Dyckerhoff \& Widmann KG y Grün \& Bilfinger AG.

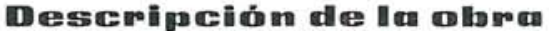

La estructura está constituida por una viga continua, simétrica, de $524,7 \mathrm{~m}$ de longitud, subdividida en siete tramos, simétricos respecto al central. Los tres tramos de acceso a partir de un estribo tienen, respectivamente, $43,44,35$ y $71 \mathrm{~m}$ de luz. 


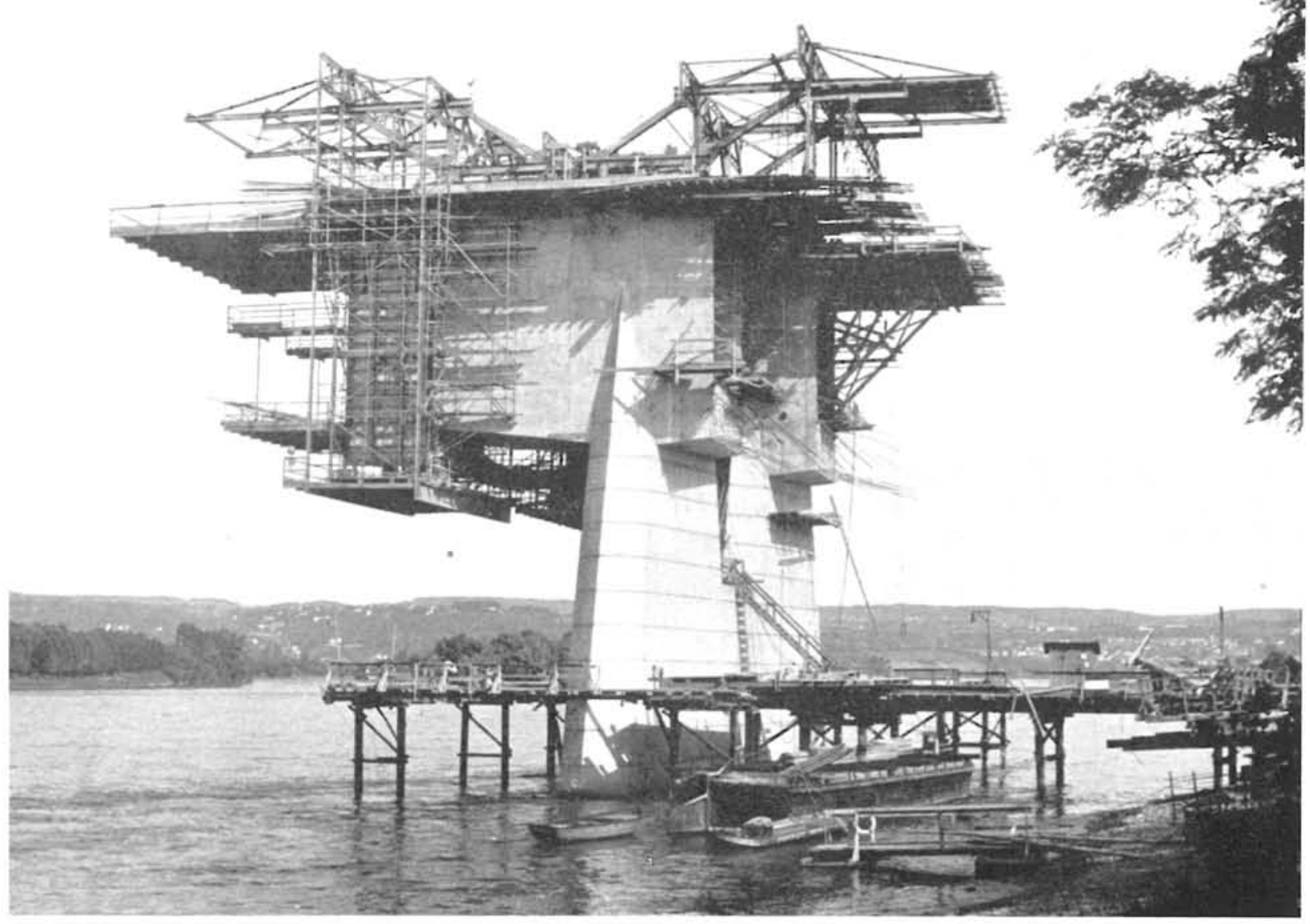

La unión de la viga con sus apoyos en el tramo central se realiza rígidamente, mientras que la de los demás se hace por medio de rodillos. En la clave del tramo central se ha previsto una articulación, la cual permite los movimientos longitudinales de la viga debidos a cambios de temperatura y deformaciones propias del puente.

Su anchura total es de 30,86 metros, dividida en dos partes independientes, cada una de las cuales presenta una sección transversal, en forma de cajón, con paredes de hormigón armado. La losa superior tiene $13,20 \mathrm{~m}$ de anchura; las dos paredes laterales son de 30 y $37 \mathrm{~cm}$ de espesor, y la losa inferior es de $7,20 \mathrm{~m}$ de anchura.

El canto de la viga sobre pilas principales es de 10,45 m, disminuyendo hasta la clave, donde tiene $3,30 \mathrm{~m}$. El espesor de la losa superior del cajón, en el tramo central, varía de $42 \mathrm{~cm}$ sobre pilas a $28 \mathrm{~cm}$ en la clave. La losa de intradós del cajón varía de $2,45 \mathrm{~m}$ en las pilas a $16 \mathrm{~cm}$

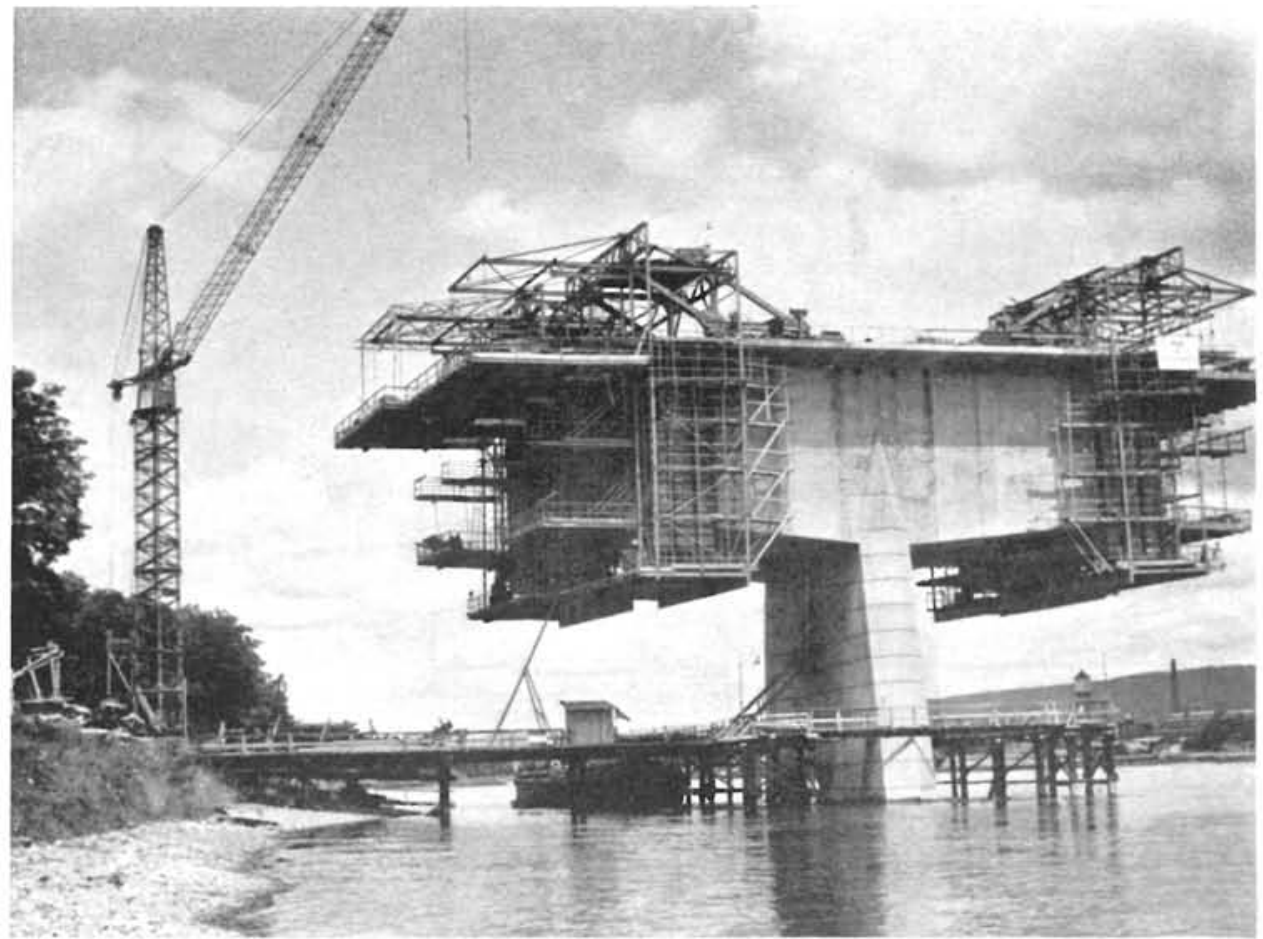




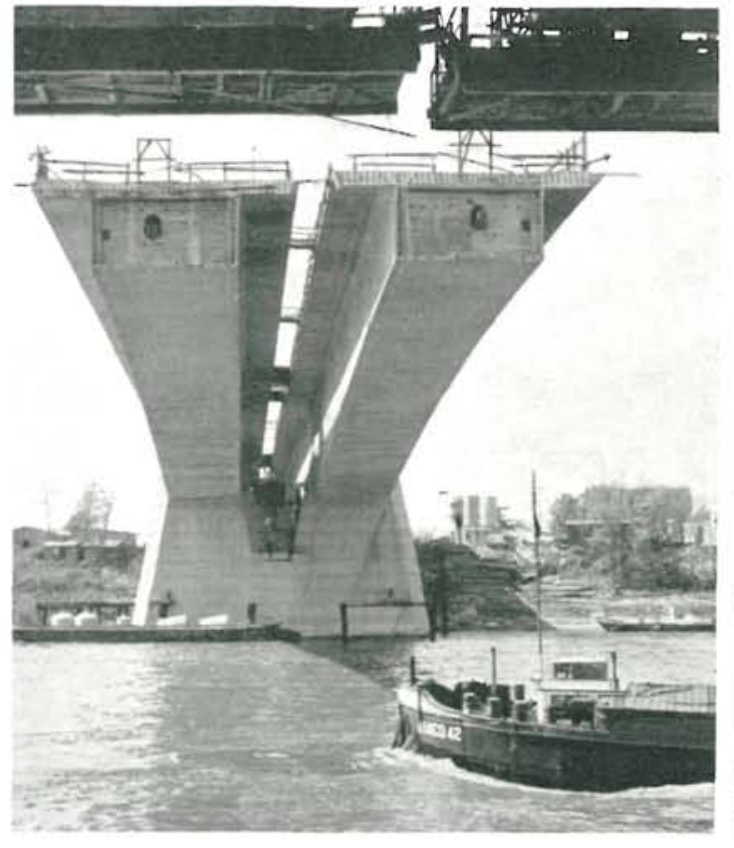

Brazo en voladizo de 40 metros.

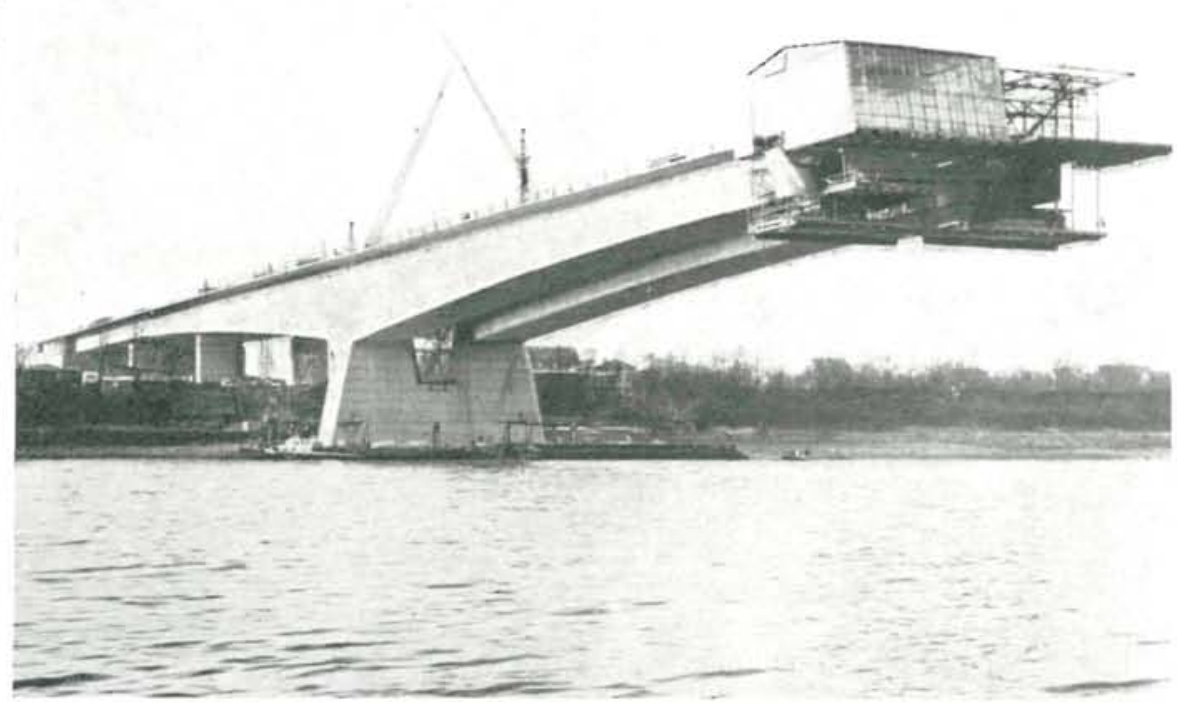

Protección del equipo de hormigonado.

en la clave. El cajón del tramo central se ha rigidizado con diafragmas transversales espaciados a 34,66 m y sólo uno, central, en los tramos de $71 \mathrm{~m}$ de luz. Para completar los andenes y el macizo central se prolongó la losa superior con voladizos laterales.

Los apoyos correspondientes a los soportes provistos de rodillos llevan una placa de Teflon, con objeto de absorber los efectos de posibles asientos en aquéllos. Todos los apoyos pueden compensarse, en caso de asientos, por medio de placas colocadas con la ayuda de gatos hidíáulicos.
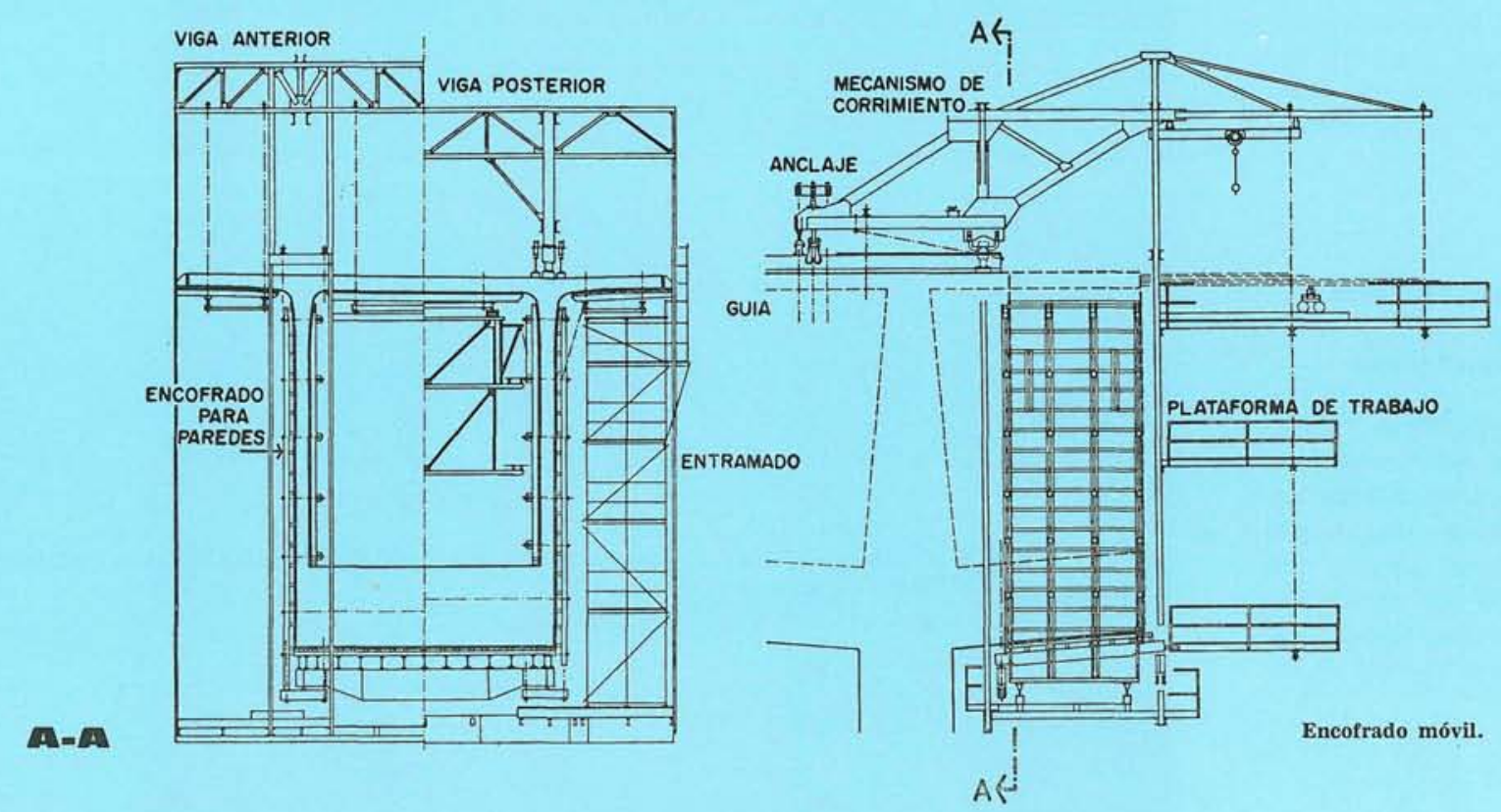
Cierre del tramo central.

Brazos equilibrados.

Tramo central cerrado.

Con objeto de evitar posibles levantamientos de la losa del tablero en los soportes del tramo central se ha colocado una capa de balasto en el interior del cajón y a la altura de cada uno de aquéllos, que son macizos, de hormigón armado y de $2,80 \mathrm{~m}$ de espesor, siendo el de los estribos de $2,5 \mathrm{~m}$ y el de los demás soportes de 1,50 m. Los laterales, cuya altura es de hasta $20 \mathrm{~m}$, se componen de dos montantes espaciados a $6,40 \mathrm{~m}$ en sentido transversal. Este último tipo de soportes cimentados a poca profundidad, dan origen a una carga máxima de 5 kilogramos $/ \mathrm{cm}^{2}$ sobre la base de cimientos. Con el fin de protegerlos contra las socavaciones, todo su perímetro está circundado por un tablestacado que desciende a $10 \mathrm{~m}$ de profundidad.

\section{Esstrabillid an al ate a estrimuteturata}

La viga continua, simétrica, se halla estáticamente indeterminada en siete puntos. Una junta longitudinal separa el puente en dos partes independientes, formadas por otros tantos cajones.
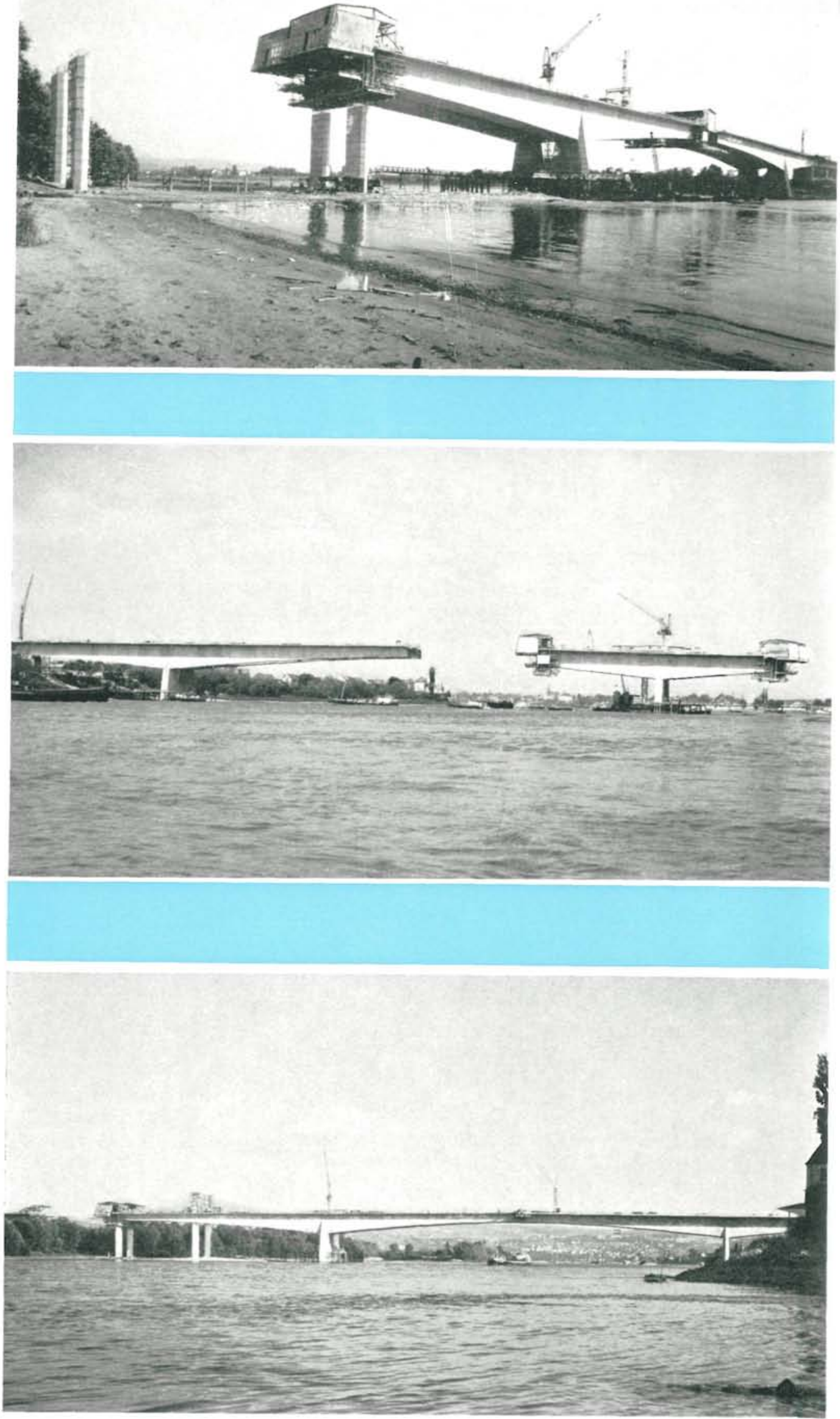
La presencia de diafragmas en el cajón permitió considerar reparticiones de cargas, de acuerdo con el principio de Saint Venan, y que la excentricidad de cargas no originase momentos secundarios de torsión importantes.

La forma trapezoidal de los soportes principales-con inclinación de $81^{\circ}$ en los lados laterales-no tiene prácticamente importancia sobre los efectos de capacidad de sustentación en la base de soportes.

La estructura se calculó de acuerdo con las normas DIN 1072, clase 60 , admitiendo un asiento de $2 \mathrm{~cm}$ en las circunstancias más desfavorables. Su comportamiento definitivo se pudo asegurar, ya que, con ayuda de gatos hidráulicos, podían reajustar las condiciones locales que se exigían en cada una de las fases constructivas. Durante el período constructivo no se rebasaron las cargas previstas para la obra terminada, lo cual permitió se pudiese construir en voladizo sin introducir parte alguna adicional.

Partiendo de los soportes principales se construyó el voladizo, a uno y otro lado-sin necesidad de apoyo intermedio-, en una longitud de $40 \mathrm{~m}$. Sin embargo, y para mayor seguridad, a partir de esta distancia se introdujo un soporte auxiliar de suspensión.

Los soportes son de hormigón armado tipo B 450. El cajón es del mismo tipo de hormigón, y el pretensado se realizó según el sistema Dywidag con barras de $32 \mathrm{~mm}$ de diámetro y acero de 80/105. La losa de tablero se pretensó en sentido transversal, y las paredes laterales, en el sentido diagonal. La losa de intradós no tiene más armaduras que las ordinarias. A la altura de los soportes principales, la losa del tablero necesitó de 560 barras de $32 \mathrm{~mm}$ para absorber un momento negativo de $200.000 \mathrm{~m} \cdot \mathrm{t}$.

Las tensiones máximas por compresión, en el hormigón de la losa de intradós, son de $150 \mathrm{~kg} / \mathrm{cm}^{2}$ en el tiempo $t_{0}$, y de $130 \mathrm{~kg} / \mathrm{cm}^{2}$, en el tiempo $t_{\infty}$. Sus armaduras experimentaron una tensión máxima de $860 \mathrm{~kg} / \mathrm{cm}^{2}$, por retracción y fluencia del hormigón. Las deformaciones plásticas dan lugar a una elevación de tensiones de compresión en el acero que llegan hasta los $2.000 \mathrm{~kg} / \mathrm{cm}^{2}$, bajando simultáneamente a 150 y hasta $130 \mathrm{~kg} / \mathrm{cm}^{2}$ la compresión en el hormigón. Las tensiones de tracción en el hormigón de las paredes son bajas y se absorben con el pretensado de barras dispuestas en diagonal.

\section{Eajomes meumáticos pama cimientos}

Como la carga en cada soporte principal es del orden de $20.000 \mathrm{t}$ y su unión con el cajón del tablero es rígida, la cimentación tenía que ser muy estable, por cuya razón se eligieron los cajones neumáticos de $34 \times 7 \mathrm{~m}$ en planta, colocados a unos $16 \mathrm{~m}$ por debajo del fondo del río. Entre la parte superior del cajón y el pie del soporte propiamente dicho hay un espesor de 4 metros.

Cada cajón tiene paredes de chapa metálica delgada-que sirve de encofrado perdido-con un peso de $50 \mathrm{t}$ y capacidad de flotación. Su hundimiento se consigue protegiéndolo de la corriente con una ataguía que sirve de guía.

Para descender dichos cajones se necesitaron presiones de $18 \mathrm{~m}$ de agua y sobrepresiones de 2 at en la cámara de trabajo, de $2,2 \mathrm{~m}$ de altura. Su colocación y descenso hasta el firme, algunas veces más profundo de lo esperado, debían de ser operaciones sucesivas, salvo en el caso de tener que suspender el hormigonado por la presencia de temperaturas muy bajas.

\section{Ejecución}

Preparado el programa de construcción de la estructura tipo cajón por trozos en voladizo, se empezó partiendo de los dos soportes principales, con ayuda de una estructura auxiliar. 

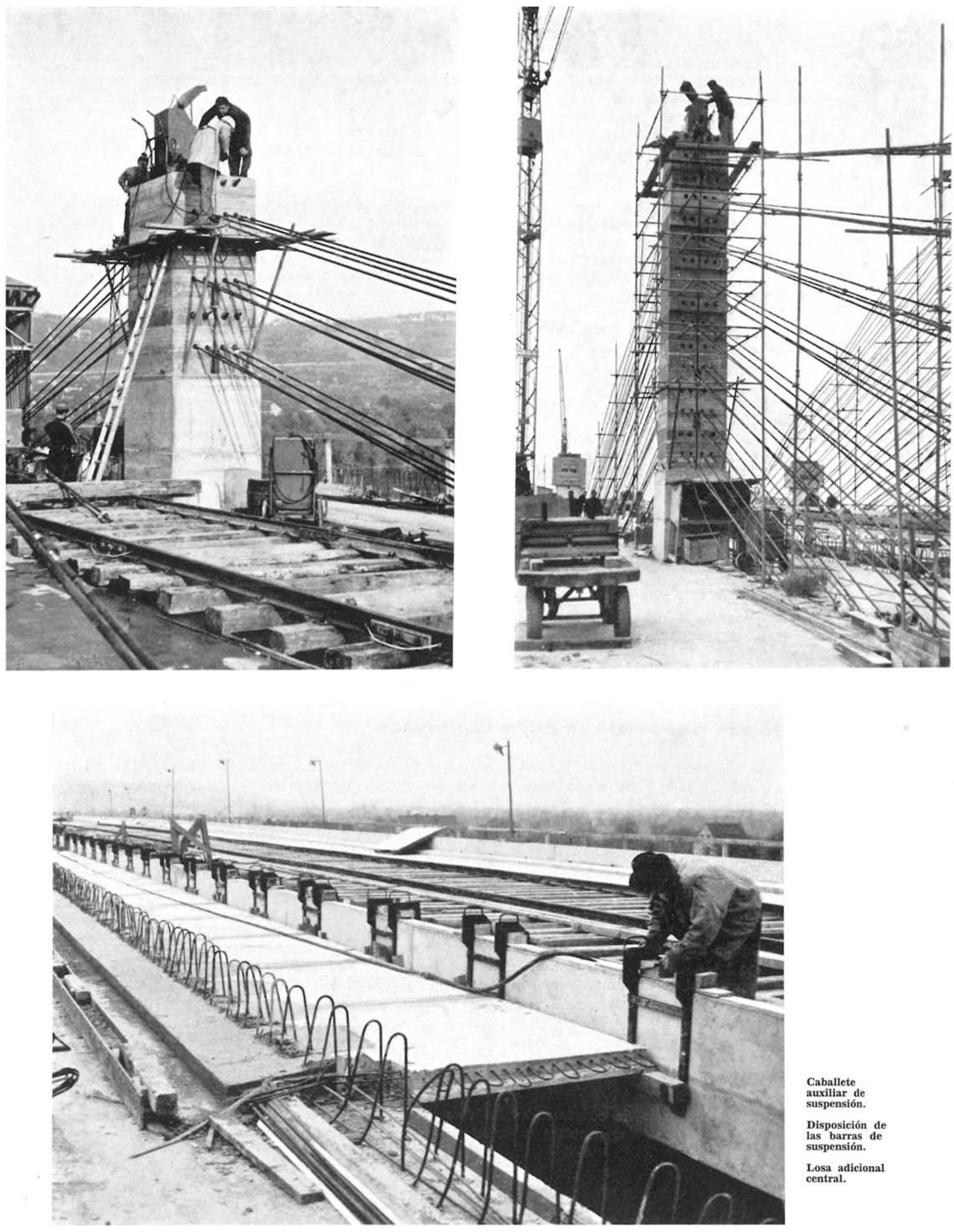

Caballete

suspensión.

Disposición de las barras de

Losa adicional central. 


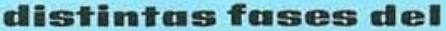

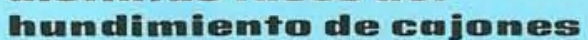

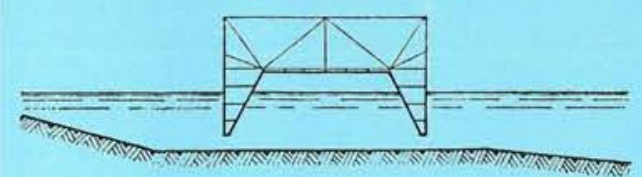

1. Hundimiento hasta el fondo.

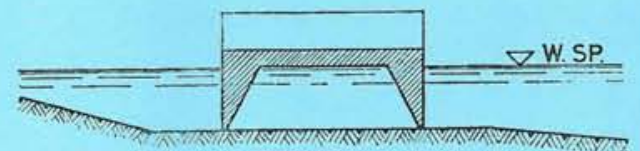

2. Hormigonado parte inferior.

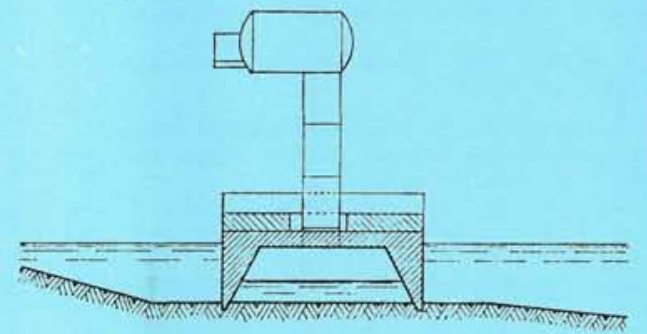

3. Instalaciones para aire comprimido.

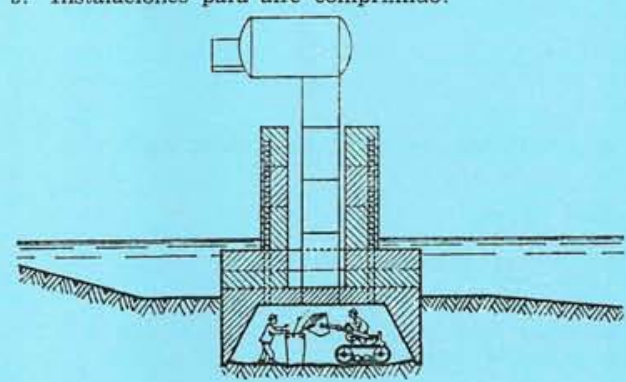

4. Descenso y hormigonado del fuste.
Se hormigonaron los 40 primeros metros a uno y otro lado de cada soporte principal y sin apoyo intermedio. Terminada esta fase se procedió al hormigonado de los otros tramos, para volver, finalmente, a continuar el hormigonado del tramo central hasta llegar al cierre en la clave. Cada semana se completaba un trozo de $7 \mathrm{~m}$ de longitud.

Como las operaciones de hormigonado se repetían continuamente, este procedimiento de construcción permitió el empleo de personal no especializado, con la consiguiente economía.

El hormigón empleado, de alta calidad, tenía una resistencia de $450 \mathrm{~kg} / \mathrm{cm}^{2}$, a los 28 días, y de $300 \mathrm{~kg} / \mathrm{cm}^{2}$, a las 30 y 33 horas, con probetas cúbicas, conseguida sin elevar excesivamente la temperatura, a fin de evitar la fisuración del hormigón que iba a quedar visto.

Después de varios ensayos se llegó a la conclusión que la temperatura inicial del hormigón tenía gran influencia en el fraguado, pues temperaturas y resistencia son funciones del tiempo. Al objeto de lograr una temperatura baja inicial se enfriaron previamente todos los componentes del hormigón, logrando así obtener una mezcla flúida a unos $15^{\circ} \mathrm{C}$.

Tiene particular importancia, en este tipo de construcciones, determinar la deformación permanente en cada uno de los trozos construidos, con objeto de introducir en ellos una contraflecha tal que, acumulándose en trozos sucesivos, permita llegar al cierre de clave en la cota prevista. En todo caso hay que tener en cuenta las distintas deformaciones que se produzcan en la estructura por efectos de asientos, torsión y fluencia.

Adaptación de J. J. Ugarte Fotost STIEBEL, WERKFOTO Y HORST GUNTHER

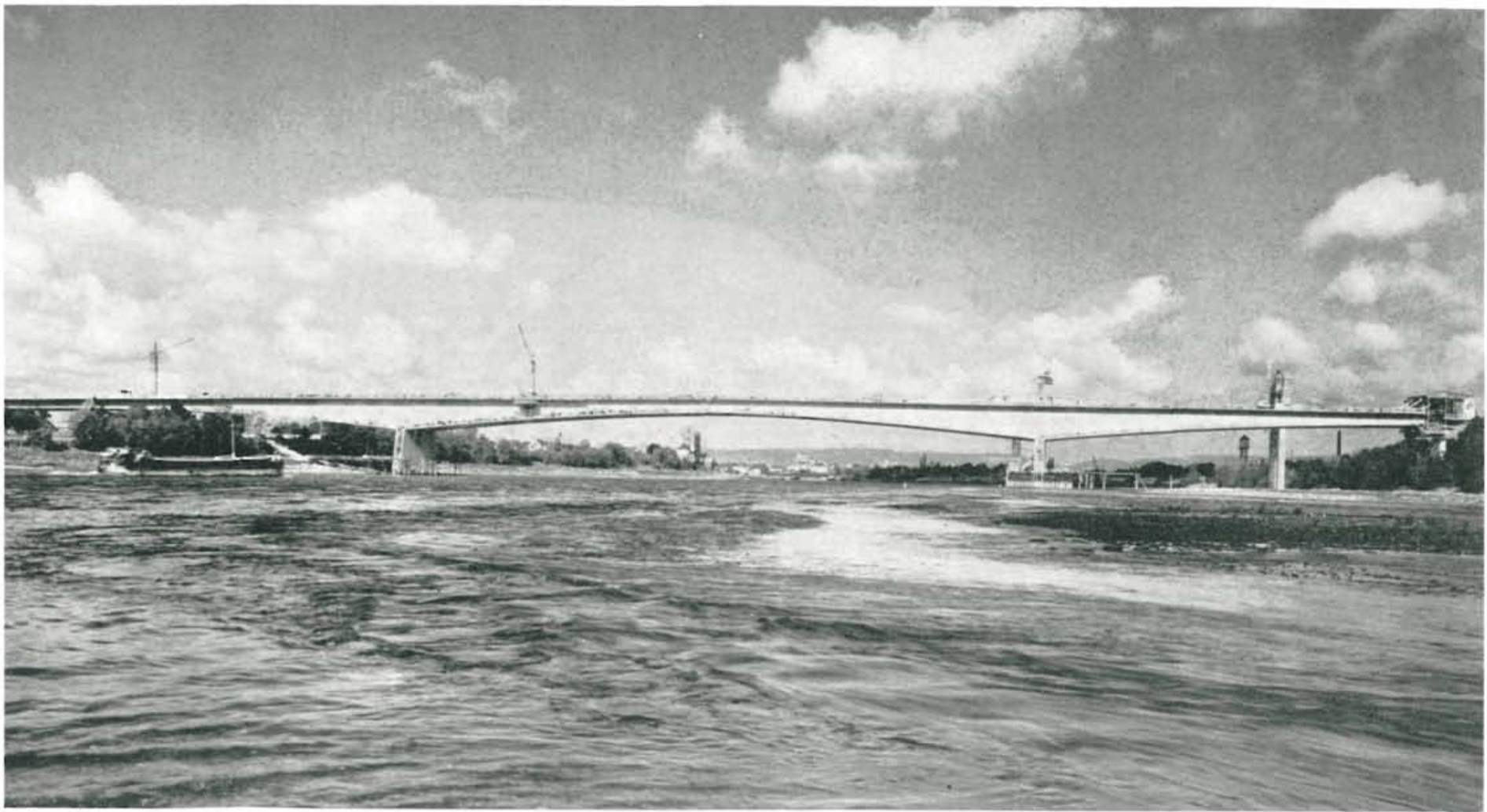




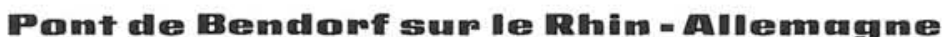

Kurt Hirschfeld, Dr. ingénieur.

Ce pont, en béton armé, récemment construit sur le Rhin, a une longueur totale de $524 \mathrm{~m}$ et a été construit selon le procédé d'encorbellements successifs, sans échafaudages auxiliaires ni cintres.

La portée de la travée centrale est de $208 \mathrm{~m}$ et les 6 autres oscillent entre 43 et $71 \mathrm{~m}$.

L'ossature du tablier se compose de deux parties indépendantes formées par une poutre caisson continue, articulée à la clef de la travée centrale et raidie à l'aide de diaphragmes transversaux espacés de 34 mètres.

Les piles centrales s'appuient sur des caissons pneumatiques qui s'enfoncent jusqu'à $16 \mathrm{~m}$, sous le lit du fleuve. La solidarisation de ces piles avec la structure caisson est rigide pour ces piles et articulée pour les autres.

La travée centrale a été construite par encorbellements successifs, sans appuis intermédiaires, sur une lon. gueur de $40 \mathrm{~m}$, distance à partir de laquelle ont été construit des appuis provisoires.

Les différents tronçons furent bétonnés et mis en contrainte à l'aide de barres de 32 mm de diamètre selon le procédé Dywidag et en tenant compte des différentes déformations expérimentées pour chacun d'eux afin d'y introduire la contre-flèche nécessaire pour fermer la travée centrale à la cote prévue.

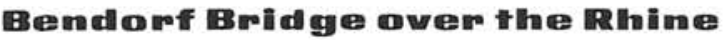

Kurt Hirschfeld, Dr. engineer.

This recently completed bridge over the Rhine is $524 \mathrm{~m}$ long, and is a reinforced concrete structure, built by successive overhangs, without the aid of formwork or scaffolding.

The centre span is $208 \mathrm{~m}$ in length, and the other six vary between 43 and $71 \mathrm{~m}$.

The deck consists of two independent parts, including a continuous box girder, with a hinge at the crown of the main span. It is stiffened with transversal diaphragms, spaced every $34 \mathrm{~m}$.

The piles rest on pneumatic caissons, which go down to $16 \mathrm{~m}$ below the river bed. The attachment between the piles and the caissons is rigid, at the central pile, and at the other piles it operates through a roller bearing.

The central span was constructed without intermediate supports for the first $40 \mathrm{~m}$, and beyond that stage, for greater safety, a provisional movable tower was built, as a temporary point of support for the free overhang.

The various sections were concreted and post-tensioned with $32 \mathrm{~mm}$ bars, by the Dywidag procedure. Account was taken of the varying deformations that took place at each section, so as to add the necessary counterdeflection that finally enabled the two sides of the arch to meet at the crown at the calculated point.

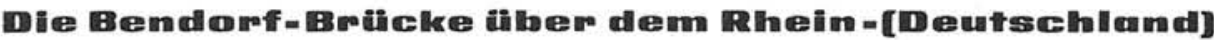

Kurt Hirschfeld, Dr. Ingenieur.

Diese kürzlich über dem Rhein erbaute Brücke hat eine Gesamtlänge von 524 Metern, besteht aus Eisenbeton und wurde nach dem Verfahren von aufeinanderfolgenden Auskragungen ausgeführt, ohne Hilfsgefüge und Lehrgerüste.

Die Lichtweite des Mittelfeldes beträgt $208 \mathrm{~m}$ und diejenige der sechs andern schwankt zwischen 43 und $71 \mathrm{~m}$.

Die Struktur der Fahrbahn besteht aus zwei unabhängigen Teilen, gebildet aus einem durchlaufenden Kastenbalken mit einem Gelenk im Scheitel des Mittelfeldes und einer Versteifung durch transversale Zwischenwände im Abstande von 34 Metern.

Die Mittelpfeiler stützen sich auf Senkkammern, welche $16 \mathrm{~m}$ unterhalb des Flussbettes eindringen. Die Verbindung zwischen diesen Pfeilern und der Kastenstruktur ist starr; bei den andern Pfeilern ist sie vom Rollentyp.

Das mittelfeld wurde auf einer Länge von $40 \mathrm{~m}$ mit aufeinanderfolgenden Auskragungen, ohne Zwischenstütze, gebaut; von dort an wurde zur Erhöhung der Sicherheit eine provisorische Hilfsstütze errichtet.

Die verschiedenen Stücke wurden mit Stangen von $32 \mathrm{~mm}$ Durchmesser betoniert und gespannt, nach dem Verfahren von Dywidag und unter Berücksichtigung der von einem jeden erlittenen verschiedenen Deformatio. nen, um die nötige Wölbung zwecks Schliessung des Mittelfeldes in der vorgesehenen Höhe zu erzielen. 